\title{
Sensor devices and biosensors in food analysis
}

\author{
Fritz Pittner
}

Received: 24 March 2009/Accepted: 24 March 2009/Published online: 21 April 2009

(C) Springer-Verlag 2009

Food is an incredibly complex mixture of mostly natural, but also artificial, substances. Thus food analysis is a very challenging field, especially when dealing with the detection of minor components, for example flavors, vitamins, antioxidants, natural toxins, more or less unwanted toxic heavy metals from soil, herbicide or pesticide residues, etc. Special clinical problems, for example metabolic defects or allergies, often result in the need to detect traces of cross contaminants, etc.

Detection of such chemically very different analytes in, for chemists, "most unfriendly surroundings" once needed very tricky and time-consuming methods, and the results obtained were often questionable. Sensor technology currently attracts increasing attention as a successor to conventional analytical techniques in the food industry. The reasons are manyfold and the potential applications widespread, including fermentation and monitoring of production processes and product quality to provide healthy, uncontaminated, high-standard food with long shelf life. For organic components, especially, the development of very selective biosensors has improved food analysis substantially. This does not, however, render careful sample preparation unnecessary and we should never forget that the quality of each result given by the readout depends on this fact.

F. Pittner $(\square)$

University of Vienna, Vienna, Austria

e-mail: Fritz.Pittner@univie.ac.at
A huge variety of chemical sensors have been invented for food analysis; many are based on electrochemical measurements but optical readout also is becoming increasingly important. Detection methods are not restricted to these two techniques only, so this special issue isrounded off by presenting various miscellaneous methods, for example piezoelectric devices with molecular imprinting.

Another very wide field is the use of immobilized antibodies, or any other biorecognitive components, on the sensor devices, selectively locking the material of interest and making it available for further sensing procedures on a transducer which can be either electrochemical or optical. Another advantage is extreme sensitivity, even down to the femtomolar range in special cases. The major drawback of biosensors is often high dependence on certain conditions necessary for biorecognition, for example $\mathrm{pH}$, temperature, or ionic strength of the analyte, a problem that can sometimes be overcome by very careful choice of immobilization techniques, which sometimes also enables reaction even in organic solvents. Biosensors are, furthermore, not restricted to analysis of naturally occurring substances-biorecognition may also be adapted to artificial additives by use of specific antibodies for recognition. Finding appropriate chemical or enzymatic reactions for electrochemical or optical monitoring can be facilitated by use of enzymes obtained from mutated or genetically engineered microorganisms. Optical monitoring combined with high sensitivity occasionally also enables miniaturization and production of microarrays, thus giving such systems a promising future.

The here compiled invited reviews and original contributions on sensor devices and biosensors in food analysis should give the reader overviews about sensor types, performance criteria, principal performance characteristics, and possible variations with respect to different analytes. 
Biochemical and physicochemical aspects of sensor setups are also discussed. A large number of references will facilitate further reading about specific topics in more depth.

The editors thank all authors for accepting our invitation to contribute to this special issue of Chemical Monthly and wish the readers a pleasant time going through the various papers and being informed about the current state of art in the field.

Fritz Pittner

Gerhard Sontag

Gisela Pittner

Issue Editors 